\title{
Studies on the growth aspects of organic L-alanine maleate: a promising nonlinear optical crystal
}

\author{
D. Balasubramanian ${ }^{1,3^{*}}$, R. Jayavel $^{2}$, P. Murugakoothan $^{3}$ \\ ${ }^{1}$ Department of Physics, Municipal School, Tiruvannamalai, India; dr.d.balu@gmail.com \\ ${ }^{2}$ Crystal Growth Centre, Anna University, Chennai, India \\ ${ }^{3}$ PG \& Research Department of Physics, Pachaiyappa's College, Chennai, India
}

Received 17 September 2009; revised 10 October 2009; accepted 12 October 2009.

\begin{abstract}
A organic nonlinear optical material, L-alanine maleate (LALM) was synthesized. Bulk Single crystals of LALM have been grown by slow cooling method with a solution $\mathrm{pH}$ of 5 . The solubility of L-alanine maleate has been determined for various temperatures. Large size single crystal of $2.0 \times 1.2 \times 0.8 \mathrm{~cm}^{3}$ has been grown with reasonable growth rate along the three crystallographic directions by optimizing the growth parameters. The structure of LALM crystal was studied by single-crystal X-ray diffraction analysis. The presence of functional groups was confirmed by Fourier transform infrared spectroscopy. The LALM crystal was analysed for its thermal and mechanical behaviours. The grown crystals have also been subjected to linear and non-linear optical property studies. From these studies, it is inferred that the LALM crystals exhibit better thermal and mechanical stabilities with improved optical properties. Thus satisfies the essential requirements for optical device fabrication.
\end{abstract}

Keywords: Organic; LALM; Crystal Growth; Bulk Single Crystals; Non-Linear Optical Crystal

\section{INTRODUCTION}

Recently, there is considerable interest in the synthesis of new nonlinear optical (NLO) material, both organic and inorganic, with large second-order optical nonlinearities, as these materials have a significant impact on laser technology, optical communication and optical storage technology etc. Over the years many organic and inorganic materials have been developed [1-4] to cover the potential applications in ultra-violet, near-and farinfrared wavelength regions. Amino acid nonlinear opti- cal materials are often formed by weak van der Waals and hydrogen bonds and hence possess high degree of delocalization. The basic structure of organic NLO materials is based on the $\pi$ bond system. Due to the overlapping of $\pi$ orbital, the delocalization of electronic charge distribution leads to a high mobility of electrons. Functionalization of both ends of the $\pi$ bond system with appropriate electron donor and acceptor groups can enhance the asymmetric electronic distribution in either or both ground and excited states, this leads to an increased optical nonlinearity. All these favourable properties paved the way for the development of amino acid crystals like L-arginine phosphate (LAP) [5], L-histidine dihydrogen phosphate (LHP) [6], L-arginine tetrafluoroborate (L-AFB) [7], L-alanine tetrafluoroborate (L-AlFB) [8], L-alanine [9], L-arginine acetate [10], and L-alanine acetate [11]. LAP crystal was reported to have promising NLO properties comparable to that of the well-known inorganic crystal of KDP. L-alanine crystal shows Type II phase matching, for doubling the Nd:YAG fundamental, by propagating the pump beam nearly normal to the $\{120\}$ and $\{011\}$ faces.

The growth of single crystals of L-alanine which is the simplest acentric member of the amino acid family has already been little investigated. In order to widen the properties of L-alanine and to develop new crystals with better NLO properties, L-alanine complexes with carboxylic acids have been tried. Maleic acid, a dicarboxylic acid with relatively large pi-conjugation has attracted much attention. Though the L-alanine maleate crystal was already grown by slow evaporation method [12], in the present study, bulk single crystals of L-alanine maleate (LALM) single crystals have been grown by the slow cooling method with optimized growth conditions first time. The grown crystals were subjected to various characterization studies. The im- 
proved optical transmittance, NLO efficiency and the mechanical stability of the grown crystals were realized.

\section{EXPERIMENTAL PROCEDURE}

\subsection{Crystal Growth}

High purity (99\%) L-alanine and analar grade maleic acid were taken in equimolar ratio and dissolved in de-ionized water. The solution was slightly heated and kept in undisturbed conditions. Three days later, transparent seed crystals were obtained. The synthesized salt was used to prepare the growth solution according to the following reaction.

$\mathrm{CH}_{3}-(\mathrm{CH})-\mathrm{NH}_{2}-\mathrm{COOH}+\mathrm{COOH}-\mathrm{CH}=\mathrm{CH}-\mathrm{COOH}$ L-alanine + Maleic acid

$\rightarrow \mathrm{CH}_{3}-(\mathrm{CH})-\mathrm{NH}_{3}^{+}-\mathrm{COOH}^{-} \mathrm{OOC}-\mathrm{CH}=\mathrm{CH}-\mathrm{COOH}$

L-alanine maleate (LALM)

The amount of L-alanine maleate dissolved in $10 \mathrm{ml}$ of water at $30^{\circ} \mathrm{C}$ was estimated from the saturated solution. The solubility was estimated for different temperatures and the solubility of L-alanine maleate at $40{ }^{\circ} \mathrm{C}$ is estimated to be $33 \mathrm{~g} / 100 \mathrm{ml}$. Crystals were grown from aqueous solution prepared from the recrystallized salt of LALM saturated at $40{ }^{\circ} \mathrm{C}$. In order to reproduce the supersaturation conditions, the solution was tested by checking the dissolution of a probe crystal over a period of one week. Then the solution was cooled down at a rate of $0.1{ }^{\circ} \mathrm{C} /$ day over a period of 25 days. Optical quality single crystal elongated in c-axis was obtained.

\subsection{Characterization}

The structure of the crystals was examined by single-crystal X-ray diffraction analysis and the lattice parameter values were determined. Powder X-ray diffraction analysis was also carried out using a Rich Seifert diffractometer with $\mathrm{CuK}_{\alpha}(\lambda=1.5418 \AA)$ radiation to verify the correctness of lattice parameter values. FTIR spectrum was recorded by the $\mathrm{KBr}$ pellet technique using a Perkin-Elmer 783 spectrophotometer in order to confirm the presence of functional groups in the crystal lattice. Optical transmittance spectrum was recorded at room temperature using Shimadzu 1601 (UV-VIS) spectrophotometer. Optical second-harmonic generation was measured for the grown crystalline sample using Kurtz and Perry technique. Thermo gravimetric (TG) and Differential thermal analysis (DTA) for L-alanine maleate dihydrate crystals were carried out by ZETZSCH-Geratebau $\mathrm{GmbH}$ Thermal Analyzer. Etching studies were carried out on the $\{011\}$ face of L-alanine maleate crystal using different etchants like water, methanol and ethanol, in order to investigate the growth mechanism and surface features. Microhardness studies were carried out on the $\{011\}$ face of the L-alanine maleate crystals.

\section{RESULTS AND DISCUSSIONS}

As-grown crystal of L-alanine maleate (LALM) is shown in Figure 1. The crystals possess well defined morphology with reasonable growth rate along all the three crystallographic directions. The molecular structure with the numbering scheme is shown in the Figure 2. The cationic alanine molecule exists with a positively charged amino group and an uncharged carboxylic acid group. The maleic acid molecule exists in the monoionized state (i.e. as a semimaleate). The semimaleate ion is essentially planar and the intramolecular hydrogen bond between atoms $\mathrm{O} 3$ and $\mathrm{O} 5$ is found to be asymmetric, as in the crystal structure of maleic acid [13]. The single crystal X- ray diffraction studies confirm the orthorhombic structure with space group $\mathrm{P} 22_{1} 2_{1} 2_{1}$. The lattice parameter values of LALM were calculated as given in the Table 1.

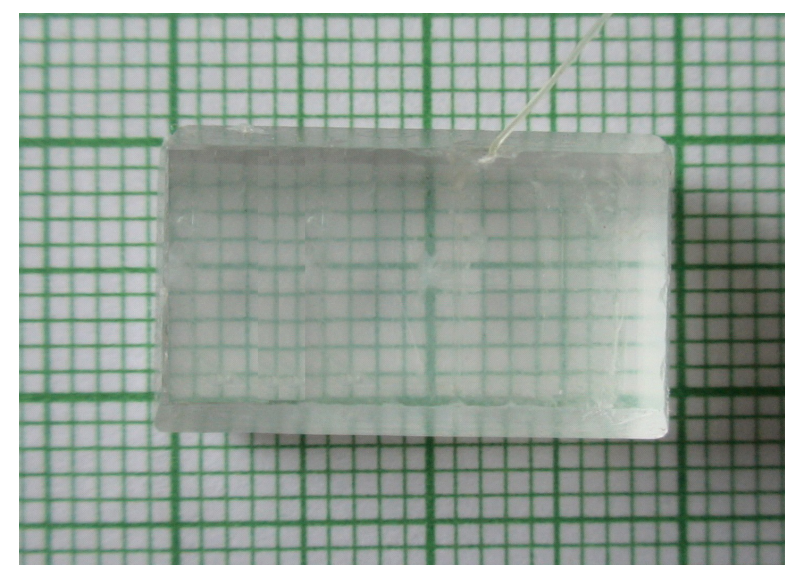

Figure 1. Bulk single crystal of LALM grown in optimizedgrowth condition.

Table 1. The single crystal X-ray data for LALM single crystal.

\begin{tabular}{|c|c|}
\hline Molecular formula & $\mathrm{C}_{3} \mathrm{H}_{8} \mathrm{O}_{2} \mathrm{~N}^{+} \cdot \mathrm{C}_{4} \mathrm{H}_{3} \mathrm{O}_{4}^{-}$ \\
\hline Molecular weight & 205.17 \\
\hline System & Orthorhompic \\
\hline Space group & $\mathrm{P} 2{ }_{1} 2_{1} 2_{1}$ \\
\hline Lattice parameter & $\begin{array}{r}\mathrm{a}=5.5873 \AA \\
\mathrm{b}=7.3864 \AA \\
\mathrm{c}=23.688 \AA\end{array}$ \\
\hline Volume (V) & $977.6 \AA^{3}$ \\
\hline Number of atom in unit cell (Z) & 4 \\
\hline Density & $1.394 \mathrm{Mgm}^{-3}$ \\
\hline
\end{tabular}


The FTIR absorption spectrum of LALM is shown in Figure 3. A broad, strong absorption in the 3300-2300 $\mathrm{cm}^{-1}$ range, including the absorptions at $3205 \mathrm{~cm}^{-1}$ corresponds to the stretching bonds of the $\mathrm{NH}_{3}{ }^{+}$ion of the amino acid. This region is due to superimposing of $\mathrm{O}-\mathrm{H}$ and $\mathrm{NH}_{3}{ }^{+}$stretching bonds. Absorption in this region was also characterized by multiple fine structures on the lower wave number side of the bond and the weak absorptions due to $\mathrm{COO}^{-}$ions. The prominent absorption band is relatively strong due to symmetric $\mathrm{NH}_{3}{ }^{+}$bending bond at $1569 \mathrm{~cm}^{-1}$. A strong band arising from $\mathrm{C}-\mathrm{COO}-$ stretching is observed at $1219 \mathrm{~cm}^{-1}$. Further strong carbonyl absorption at $1722 \mathrm{~cm}^{-1}$ confirms the $\mathrm{COOH}$ and $\mathrm{COO}^{-}$groups of the compound. The $\mathrm{C}=\mathrm{O}$ stretch of carboxylic acid was observed to produce its peak in the same region of asymmetrical $\mathrm{NH}_{3}{ }^{+}$bending vibration.

The $\mathrm{CH}_{3}$ bending modes were assigned to the peak at $1374 \mathrm{~cm}^{-1}$. O-H bending of the $\mathrm{COOH}$ group was observed at $1333 \mathrm{~cm}^{-1}$. The peaks between 918 and 1106 $\mathrm{cm}^{-1}$ were assigned to asymmetrical coupled vibration of maleic acid and alanine.

This analysis also indicates that the protonation of carboxyl group in alanine takes place by maleic acid. The absorptions of LALM have been compared with those of the L-alanine [14] in Table 2. The shifts in the positions of the characteristic peaks confirm the formation of the compound. a<smiles>CC(C)C(=O)O</smiles>

b

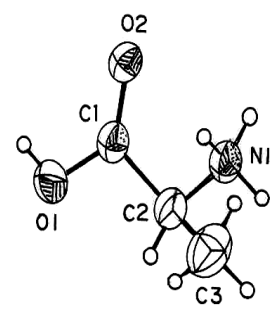<smiles>O=C(O)C=CC(=O)O</smiles>

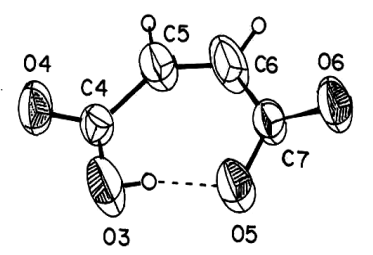

Figure 2. The molecular structure of LALM with atomnumbering scheme and 50\%probability displacement ellipsoids.

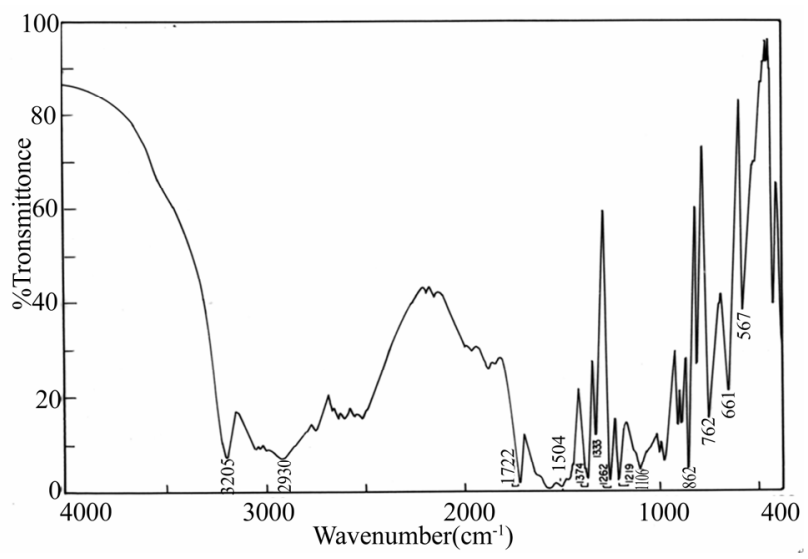

Figure 3. FT-IR spectrum of LALM crystal.

Table 2. FT-IR spectral band assignments of LALM.

\begin{tabular}{ccl}
\hline L-alanine & LALM & \multicolumn{1}{c}{ Assignments } \\
\hline & 3205 & $\mathrm{NH}_{3}{ }^{+}$asymmetric stretching \\
& 2930 & $\mathrm{C}-\mathrm{H}$ stretching \\
& 1722 & $\mathrm{COO}^{-}$stretching \\
1506 & 1504 & $\mathrm{NH}_{3}{ }^{+}$symmetric bending \\
1361 & 1374 & $\mathrm{C}^{-}$deformation in $\mathrm{CH}_{3}$ \\
& 1333 & $\mathrm{O}-\mathrm{H}$ plane deformation in $\mathrm{COOH}$ \\
& 1262 & $=\mathrm{C}-\mathrm{H}$ deformation (over tone) \\
& 1219 & $\mathrm{C}-\mathrm{COO}^{-}$stretching \\
1114 & 1106 & $\mathrm{C}-\mathrm{O}$ stretching, $\mathrm{NH}_{3}$ rocking \\
850 & 862 & $\mathrm{O}-\mathrm{H}$ out-of-plane deformation \\
772 & 762 & $\mathrm{CH}$ rocking \\
649 & 661 & O-C = O in plane deformation \\
& 567 & $\mathrm{COO}^{-}$wagging \\
\hline
\end{tabular}

The transmission spectrum of LALM crystal was recorded in the range 200-1200 nm is shown in Figure 4. A sample of thickness $2 \mathrm{~mm}$ was used to record the transmission spectrum. The crystal possesses $75 \%$ transmittance and the lower cutoff is found to be as low as $320 \mathrm{~nm}$, allowing for frequency conversion down to UV-region which account for the suitability of this material for optoelectronics applications and the second and third harmonic generation of $\mathrm{Nd}$ :YAG fundamental.

The powder second harmonic generation (SHG) test was carried out for LALM using Kurtz and Perry technique. Powdered sample of LALM was tightly packed in the micro capillary tubes of uniform diameter $(1.5 \mathrm{~mm})$ and irradiated by an incident laser radiation $1064 \mathrm{~nm}$ of pulse width $8 \mathrm{~ns}$ and pulse energy of 10-800 $\mathrm{mJ}$ from a Q-switched quanta ray of Nd:YAG laser. KDP was used for calibrating the SHG intensity. The second harmonic nonlinearity of LALM was confirmed by the emission of green radiation $(532 \mathrm{~nm})$ by the crystal. The powder SHG efficiency of LALM was found to be 1.2 times that of the standard KDP. This confirms that the LALM has higher SHG efficiency than the relative efficiencies of L-alanine $(0.2)$ and L-alanine acetate $(0.3)$ with respect to $\mathrm{KDP}[15]$. 


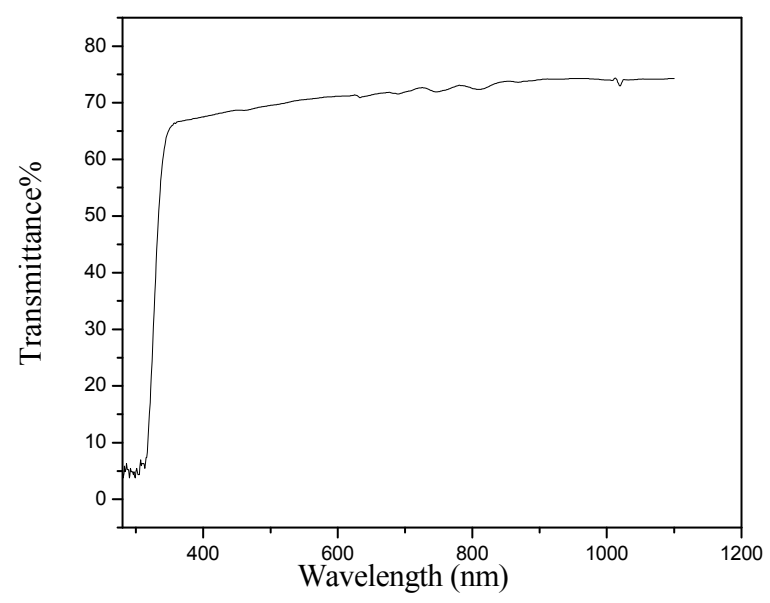

Figure 4. Optical transmittance spectrum of LALM crystal.

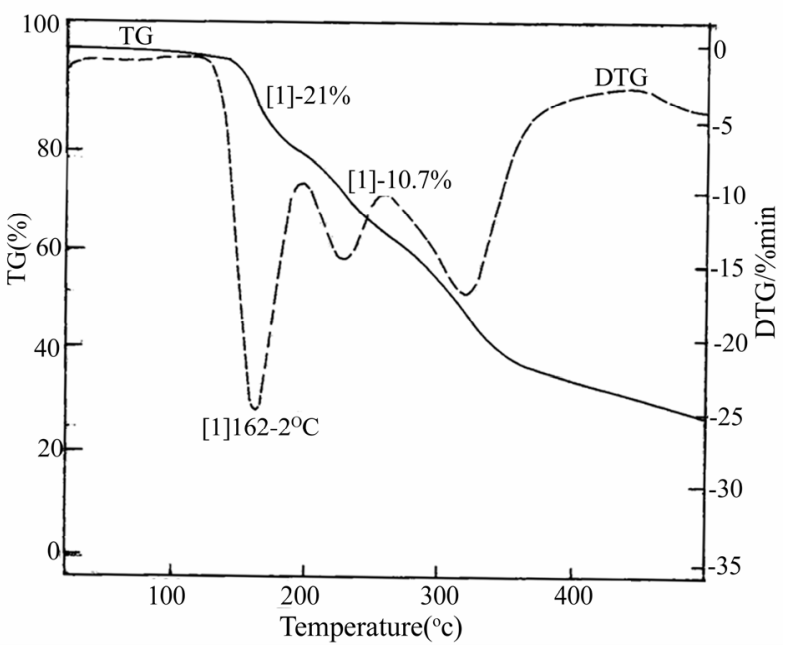

(a)

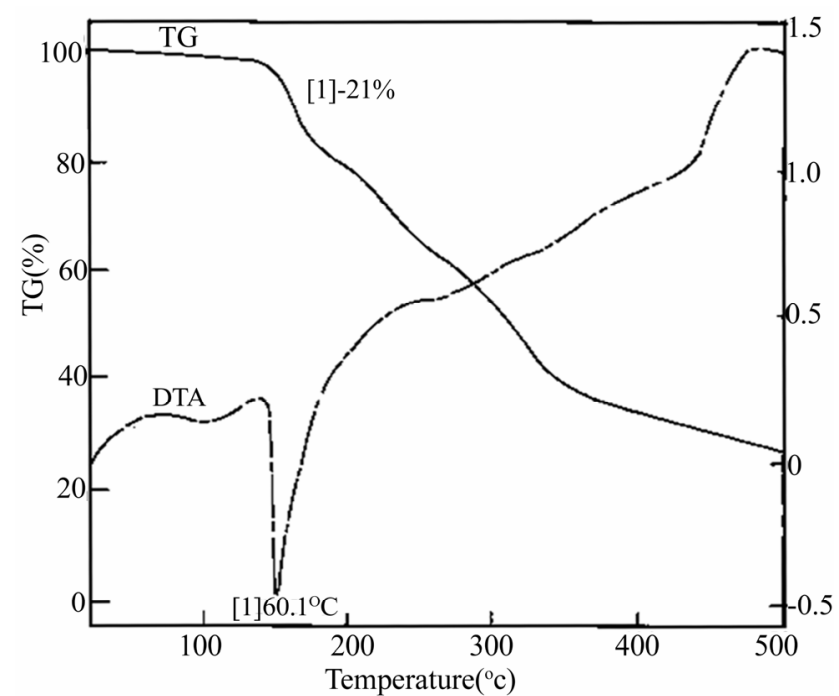

(b)

Figure 5. (a) TG / DTG curves of LALM; (b) DTA/ TG curves of LALM.

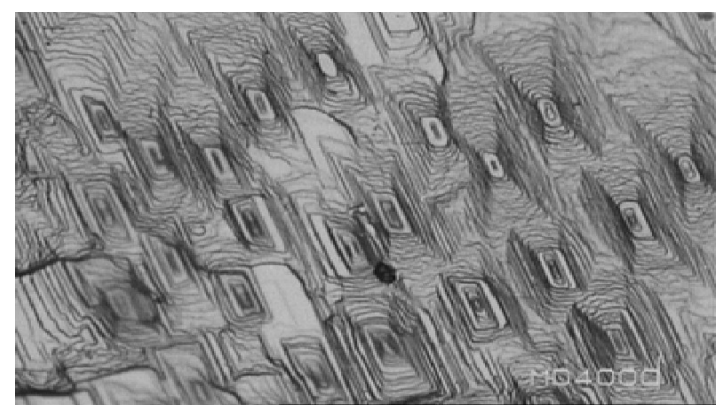

(a)

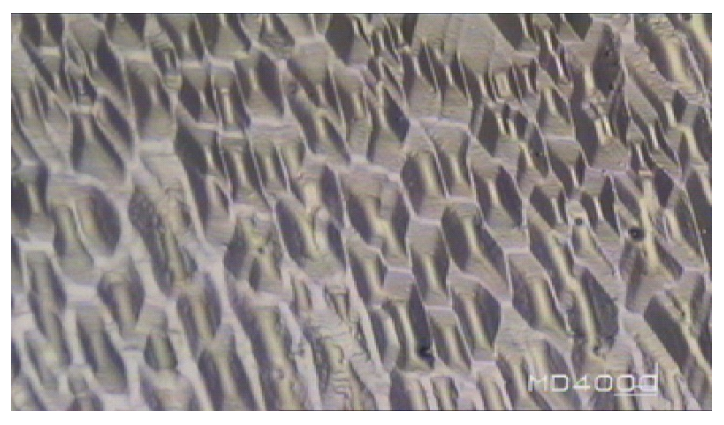

(b)

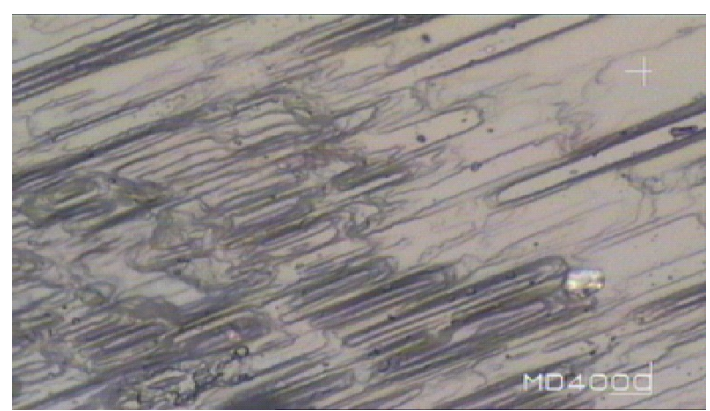

(c)

Figure 6. Etch patterns obtained on (011) of LALMcrystal for different etchants. (a) Water; (b) Methanol ; (c) Ethanol.

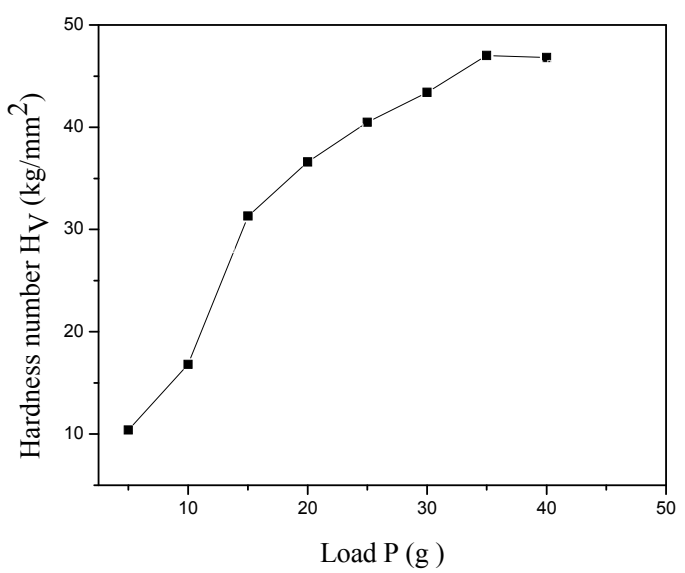

Figure 7. Load (P) Vs Hardness number (Hv) of LALM crystal. 
Simultaneous thermo-gravimetric analysis (TG) and differential thermal analysis (DTA) were carried out for the as-grown LALM crystals to study the thermal stability. The characteristic curves are shown in Figure 5a and 5b. Finely powdered crystal was used for the TG/DTA analysis in the temperature range of 26 to $500{ }^{\circ} \mathrm{C}$ with a heating rate of $5^{\circ} \mathrm{C} / \mathrm{min}$. The alumina $\left(\mathrm{Al}_{2} \mathrm{O}_{3}\right)$ crucible was used as a reference for the sample. A weight loss of $21 \%$ occur at $162{ }^{\circ} \mathrm{C}$ in TGA corresponds to the decomposition range of LALM. An endothermic peak observed at $160{ }^{\circ} \mathrm{C}$ in DTA is attributed to the utilization of thermal energy to overcome the valence bonding between the alaninium cation and the maleate anion, which happens during the initial stage of decomposition. As the temperature is increased further, maleic acid decomposes and becomes anhydride and results in the further release of $\mathrm{CO}_{2}$ and $\mathrm{CO}$ molecules at 205 and $258{ }^{\circ} \mathrm{C}$, which is evident from the DTG. The reactions of simplest amino acids induced by heating include the condensation reactions of carboxyl and amino groups leading to the formation of peptide bonds. In the dehydration at the initial stage, $\mathrm{H}_{2} \mathrm{O}$ molecule is not liberated immediately; instead, it is absorbed by alumina, which acts as a catalyst, and then is released along with another water molecule obtained from the decomposition of alanine at $320^{\circ} \mathrm{C}$. Because of this, an endothermic effect is noted in DTA. $\mathrm{CO}$ and $\mathrm{CH}_{4}$ molecules are liberated at around $450^{\circ} \mathrm{C}$.

The NLO efficiency of the grown crystals mainly depends on their optical quality, because the segregated impurities and dislocations occurring during the growth results in the distortion of the optical beam to be processed. In the present investigation, grown crystal of LALM was subjected to chemical etching to study the microstructural imperfection or crystal defects in the grown crystal. A thin plates of $3 \mathrm{~mm}$ thickness parallel to $\left\{\begin{array}{lll}0 & 1 & 1\end{array}\right\}$ face were cut from the as grown crystal of LALM with the help of a wet thread. Polishing of the surfaces was carried out using soft felt-cloth wetted with ethanol and tertiary butanol mixture $(3: 1)$. Polished plates of $2 \mathrm{~mm}$ in thickness, free from visible inclusions or cracks were selected for etch pit study. Etchants employed to reveal dislocations are taken in homologous series of alcohols i.e. water, methanol and ethanol. Etching of the surfaces was carried out by dipping the plates in etchants for few seconds to few minutes at room temperature and then wiping them with dry filter paper. Etch patterns were observed and photographed under an optical (Carl-Zesis Jenavert) microscope in the reflected light. Elongated circular etch pits were observed when LALM single crystal was etched with water for five seconds as shown in Figure 6a. There was no change in etch pit dimension and density with varying etching time (10-20 s). Trapezoid etch pits were observed as shown in Figure $\mathbf{6 b}$ when LALM single crystal was etched with methanol for five seconds. Rectan- gular etch pits were observed when the crystal was etched with ethanol for fifteen seconds as shown in Figure 6c. From the results of etching behavior of different etchants on LALM crystals, it is inferred that all the organic solvents used in this experiment have successfully revealed the presence of dislocation in the crystal. The observed etch pits, due to layer growth, confirmed the two dimensional (2D) nucleation mechanism with less dislocations. Fast dissolving etchant like water produces better contrasting dislocation etch pits of all surfaces and hence it is intensive to surfaces orientation.

Hardness value on the (011) of LALM crystal was estimated for different loads. The relation between hardness number (Hv) vs load (P) for LALM is shown in Figure 7. At lower loads, hardness is relatively lower and it increases for higher loads and remains constant up to $40 \mathrm{~g}$. Above $40 \mathrm{~g}$, a significant cracking occurred due to the release of internal stress generated locally by indentation. It has been observed that the hardness values of LALM are comparable with pure L-alanine.

\section{CONCLUSIONS}

A new organic optical material for second order NLO applications, L-alanine maleate (LALM) was synthesized. Single crystals were grown and characterized by X-ray diffraction (single crystal XRD) to confirm the formation of the crystalline phase. FT-IR spectroscopic analysis confirms the presence of all the functional groups in the crystal lattice. Etching studies were carried out for LALM crystal using various etchants. Mechanical behavior of grown crystal was studied on (011) using micro hardness measurement and the hardness values are found to be comparable with pure L-alanine. TG-DTA studies reveal that the material starts decomposing at 162.2 ${ }^{\circ} \mathrm{C}$. The UV-Vis spectrum establishes the good transmittance window and the lower cutoff are found to be as low as $320 \mathrm{~nm}$, allowing for frequency conversion down to UV-region. From the Kurtz-Perry powder technique, the second harmonic generation efficiency of the grown LALM crystal was found to be 1.2 times that of KDP crystal.

\section{REFERENCES}

[1] N. B. Singh, T. Henningsen, R. H. Hopkins, R. Mazelsky, R. D. Hamacher, E. P. Supertzi, F. K. Hopkins, D. Zelmon, and E. O. P. Singh, (1993) Nonlinear optical characteristics of binary organic system. J. Cryst. Growth, 128, 976-980.

[2] D. S. Chemla and J. Zyss, (1987) Nonlinear optical properties of organic molecules and crystals. $1 \& 2$, Academic Press, New York.

[3] J. Badan, R. Hierle, A. Perigaud, J. Zyss, and D. J. Williams, (1993) Nonlinear optical properties of organic 
molecules and polymeric materials. American Chemical Society, Washington, DC, American Chemical Sym- posium Series, 233

[4] R. Rajasekaran, P. M. Ushasree, R. Jayavel, and P. Ramasamy, (2001) Growth and characterization of zinc thiourea chloride (ZTC): A semiorganic nonlinear optical crystal. J. Cryst. Growth, 229, 563-567.

[5] D. Xu, M. Jiang, and Z. Tan, (1983) A new phase matchable nonlinear optical crystal L-arginine phosphate monohydrate, Acta Chem. Sinica, 41, 570-573.

[6] E. Espinosa, B. Wyncke, F. Bréhat, X. Gerbaux, S. Veintemillas, and E. Molins, (1997) Infrared vibrational spectra of L-histidinium dihydrogen orthophosphate orthophosphoric acid (LHP). Infrared Physics \& Technology, 38, 449-458.

[7] D. R. Babu, D. Jayaraman, R. M. Kumar, G. Ravi, and R. Jayavel, (2003) Growth aspects of semi-organic nonlinear optical L-arginine tetrafluoroborate single crystals, Journal of Crystal Growth, 250, 157-161.

[8] D. R. Babu, D. Jayaraman, R. M. Kumar, and R. Jayavel, (2002) Growth and characterization of non-linear optical L-alanine tetrafluoroborate (L-AlFB) single crystals, J. Crystal Growth, 245, 121-125.

[9] C. Razzetti, M. Ardoino, L. Zanotti, M. Zha, and C. Pao- rici, (2002) Solution growth and characterisation of 1-alanine single crystals. Cryst. Res. Technol, 37, 456465.

[10] R. Muralidharan, R. Mohankumar, R. Jayavel, and P. Ramasamy, (2003) Growth and characterization of L-arginine acetate single crystals: A new NLO material. J. Cryst. Growth, 259, 321-325.

[11] R. M. Kumar, D. R. Babu, D. Jayaraman, R. Jayavel, and K. Kitamura, (2005) Studies on the growth aspects of semi-organic l-alanine acetate: A promising NLO crystal, J. Crystal Growth, 275, 1935-1939.

[12] S. A. Natarajan, S. M. Britto, and E. Ramachandran (2006) Growth, thermal, spectroscopic, and optical studies of 1-alaninium maleate, a new organic nonlinear optical material. Crystal Growth \& Design, 6(1), 137-140.

[13] M. N. G. James and G. J. B. Williams, (1974) A refinement of the crystal structure of Maleic Acid. Acta Cryst. B30, 1249-1257.

[14] K. Nakamoto, (1970) Infrared spectra of inorganic and coordination compounds. Wiley-Interscience, New York, 233.

[15] D. R. Babu, (2003) Ph.D Thesis, University of Madres, India. 